\title{
Delay-Dependent Stabilization of Stochastic Interval Delay Systems with Nonlinear Disturbances
}

\author{
Guoliang $\mathrm{Wei}^{a}$, Zidong Wang ${ }^{a, b, *}$, Huisheng $\mathrm{Shu}^{c}$ and Jian'an Fang ${ }^{a}$
}

\begin{abstract}
In this paper, a delay-dependent approach is developed to deal with the robust stabilization problem for a class of stochastic time-delay interval systems with nonlinear disturbances. The system matrices are assumed to be uncertain within given intervals, the time delays appear in both the system states and the nonlinear disturbances, and the stochastic perturbation is in the form of a Brownian motion. The purpose of the addressed stochastic stabilization problem is to design a memoryless state feedback controller such that, for all admissible interval uncertainties and nonlinear disturbances, the closed-loop system is asymptotically stable in the mean square, where the stability criteria are dependent on the length of the time delay and therefore less conservative. By using the Itô's differential formula and the Lyapunov stability theory, sufficient conditions are first derived for ensuring the stability of the stochastic interval delay systems. Then, the controller gain is characterized in terms of the solution to a delay-dependent linear matrix inequality (LMI), which can be easily solved by using available software packages. A numerical example is exploited to demonstrate the effectiveness of the proposed design procedure.
\end{abstract}

\section{Keywords}

Robust stabilization; stochastic interval systems; linear matrix inequality; nonlinear disturbance; delay-dependent criteria

\section{INTRODUCTION}

Interval systems have been well known for their importance in practical applications. When modeling realtime plants, the parameter uncertainties are unavoidable, which would lead to perturbations of the elements of a system matrix in a state-space model. These uncertainties may arise from variations of the operating point, aging of the devices, identification errors, etc. As a result, the parameters of a system matrix are estimated only within certain closed intervals. In recent years, the stability analysis and stabilization problems of various deterministic interval systems have received considerable research attention, see e.g. [11,14] and the references therein. Very recently, in [13], the stability analysis problem of a class of stochastic delay interval systems has been considered by using the Razumikhin method.

In view of time delays being commonly residing in practical systems, the past few decades have witnessed significant progress on filtering and control for linear/nonlinear systems with various types of delays, and a large amount of literature has appeared on the general topic of time-delay systems, see e.g. $[1,6,7,15-17,20$, 22, 23]. In particular, the linear matrix inequality (LMI) technique has been extensively used because of its computational efficiency, and a great number of LMI-based results have been published, see e.g. [2, 18, 19].

This work was supported in part by the Engineering and Physical Sciences Research Council (EPSRC) of the U.K. under Grant GR/S27658/01, the Nuffield Foundation of the U.K. under Grant NAL/00630/G, and the Alexander von Humboldt Foundation of Germany.

${ }^{a}$ School of Information Sciences and Technology, Donghua University, Shanghai 200051, China.

${ }^{b}$ Department of Information Systems and Computing, Brunel University, Uxbridge, Middlesex, UB8 3PH, United Kingdom.

${ }^{c}$ Department of Applied Mathematics, Donghua University, Shanghai 200051, China.

${ }^{*}$ Corresponding author. Email: Zidong.Wang@brunel.ac.uk, Fax: ++44/1895 251686. 
It is worth mentioning that, since delay-dependent LMI techniques take into account the information on the length of delays, delay-dependent stability criteria tend to be less conservative than the traditionally delayindependent ones especially when the time delays are known and small, see [2-5,25,27,28]. Moreover, some improved delay-dependent techniques has recently been developed, see e.g. [8-10,24] for some up-to-date results.

In real-time systems, the signal transmission is usually a noisy process brought on by random fluctuations from probabilistic causes and, therefore, stochastic modeling has been of vital importance in many branches of science such as biology, economics and engineering applications. Recently, many fundamental results for deterministic systems have been extended to stochastic systems. The robust stability, stabilization, control and filtering problems for stochastic systems have been investigated by many researchers, and a lot of results on these topics have been reported in the literature, see e.g. [2, 18,21,26]. It is noticed that the delaydependent technique has been applied to the analysis and synthesis of stochastic systems in, for example, [3,27]. Unfortunately, up to now, the stability analysis and stabilization problems for stochastic time-delay interval systems with nonlinear disturbances have not been adequately addressed by delay-dependent technique yet, which remains as an interesting research topic.

In this paper, we deal with the robust stability and stabilization problems for a class of stochastic timedelay interval systems with nonlinear disturbances by developing delay dependent analysis techniques. The robust stability analysis problem is first dealt with, where the aim is to derive sufficient conditions such that the system is asymptotically stability in the mean square, dependent on the length of the time delay, for all admissible nonlinear disturbances as well as intervally varying uncertain parameters. Then, we tackle the robust stabilization problem where a memoryless state feedback controller is designed to stabilize the closedloop system. By using Itô's differential formula and the Lyapunov stability theory, sufficient conditions for the solvability of these problems are derived in term of linear matrix inequalities, which can be easily checked by resorting to available software packages. A numerical example is exploited to demonstrate the effectiveness of the results obtained.

Notation In this paper, $\mathbb{R}^{n}$ and $\mathbb{R}^{n \times m}$ denote, respectively, the $n$ dimensional Euclidean space and the set of all $n \times m$ real matrices. $L_{2}[0, \infty)$ is the space of square-integrable vector functions over $[0, \infty)$. $|\cdot|$ refers to the Euclidean norm in $\mathbb{R}^{n}$, and $\|\cdot\|_{2}$ stands for the usual $L_{2}[0, \infty)$ norm. We let $\tau>0, C\left([-\tau, 0] ; \mathbb{R}^{n}\right)$ denote the family of continuous functions $\phi$ from $[-\tau, 0]$ to $\mathbb{R}^{n}$ with the norm $\|\phi\|=\sup _{-\tau \leq \theta \leq 0}|\phi(\theta)|$, and $I$ denote the identity matrix of compatible dimension. The notation $X \geq Y$ (respectively, $X>Y$ ) where $X$ and $Y$ are symmetric matrices, means that $X-Y$ is positive semi-definite (respectively, positive definite). For a matrix $M, M^{T}$ represents its transpose, $\lambda_{\max }(M)$ (respectively, $\lambda_{\min }(M)$ ) stands for its maximum (respectively, minimum) eigenvalue and its operator norm is denoted by $\|M\|=\sup \{|M x|:|x|=1\}=$ $\sqrt{\lambda_{\max }\left(M^{T} M\right)} .\left(\Omega, \mathcal{F},\left\{\mathcal{F}_{t}\right\}_{t \geq 0}, \mathcal{P}\right)$ is a complete probability space with a filtration $\left\{\mathcal{F}_{t}\right\}_{t \geq 0}$ satisfying the usual conditions (i.e., the filtration contains all $P$-null sets and is right continuous). Denote by $L_{\mathcal{F}_{0}}^{p}\left([-h, 0] ; \mathbb{R}^{n}\right)$ the family of all $\mathcal{F}_{0}$-measurable $C\left([-\tau, 0] ; \mathbb{R}^{n}\right)$-valued random variables $\xi=\{\xi(\theta):-\tau \leq \theta \leq 0\}$ such that $\sup _{-\tau \leq \theta \leq 0} \mathbb{E}|\xi(\theta)|^{p}<\infty$, where $\mathbb{E}\{x\}$ stands for the expectation of stochastic variable $x$. The shorthand $\operatorname{diag}\left\{M_{1}, \ldots, M_{n}\right\}$ denotes a block diagonal matrix with diagonal blocks being the matrices $M_{1}, \ldots, M_{n}$. The notation $\mathfrak{M}_{n}\left[\left(M_{1}\right)_{i_{1}, j_{1}},\left(M_{2}\right)_{i_{2}, j_{2}}, \ldots,\left(M_{r}\right)_{i_{r}, j_{r}}\right]$ denotes a $n t h$-order block square matrix whose all nonzero blocks are the $i_{1} j_{1}$ th block $M_{1}$, the $i_{2} j_{2}$ th block $M_{2}, \ldots$, the $i_{r} j_{r}$ th block $M_{r}$, and all other blocks are zero matrices. In symmetric block matrices, the symbol $*$ is used as an ellipsis for terms induced by symmetry . Matrices, if not explicitly stated, are assumed to have compatible dimensions. 


\section{Problem Formulation}

For a matrix $D_{n_{1} \times n_{2}}$, define the following matrix interval:

$$
D_{I}=[\underline{D}, \bar{D}]=\left\{D=\left[d_{i j}\right]_{n_{1} \times n_{2}}: \underline{\mathrm{d}}_{i j} \leq d_{i j} \leq \bar{d}_{i j}, 1 \leq i \leq n_{1}, 1 \leq j \leq n_{2}\right\},
$$

where $\underline{\mathrm{D}}=\left[\underline{\mathrm{d}}_{i j}\right]_{n_{1} \times n_{2}}$ and $\bar{D}=\left[\bar{d}_{i j}\right]_{n_{1} \times n_{2}}$ satisfy $\underline{\mathrm{d}}_{i j} \leq \bar{d}_{i j}$ for all $1 \leq i \leq n_{1}, 1 \leq j \leq n_{2}$.

Consider the following stochastic time-delay interval system with nonlinear disturbance:

$$
\begin{aligned}
d x(t) & =\left[A x(t)+A_{d} x(t-\tau)+B u(t)+f(x(t), x(t-\tau))\right] d t+E x(t) d \omega(t) \\
x(t) & =\phi(t), \quad \forall t \in[-\tau, 0]
\end{aligned}
$$

where $x(t) \in \mathbb{R}^{n}$ is the state, $u(t) \in \mathbb{R}^{p}$ is the control input, $f(\cdot, \cdot)$ is an unknown nonlinear exogenous disturbance input, $\omega(t)$ is a one-dimensional Brownian motion satisfying

$$
\mathbb{E}\{d \omega(t)\}=0, \quad \mathbb{E}\left\{d \omega^{2}(t)\right\}=d t .
$$

Furthermore, $\tau$ is a real constant time delay satisfying $0 \leq \tau<\infty$, and $\phi(t) \in C\left([-\tau, 0] ; \mathbb{R}^{n}\right)$ is the initial function. The system matrices $A \in A_{I}, A_{d} \in A_{d I}, B \in B_{I}$, and $E \in E_{I}$, where $A_{I}=[\underline{A}, \bar{A}]=\left\{A=\left[a_{i j}\right]_{n \times n}\right\}$, $A_{d I}=\left[\underline{\mathrm{A}}_{d}, \bar{A}_{d}\right]=\left\{A_{d}=\left[a_{d i j}\right]_{n \times n}\right\}, B_{I}=[\underline{\mathrm{B}}, \bar{B}]=\left\{B=\left[b_{i j}\right]_{n \times p}\right\}$, and $E_{I}=[\underline{\mathrm{E}}, \bar{E}]=\left\{E=\left[e_{i j}\right]_{n \times n}\right\}$.

By setting

$$
\begin{aligned}
A_{0} & =\frac{1}{2}(\underline{\mathrm{A}}+\bar{A}), \quad \tilde{A}=\left(\tilde{a}_{i j}\right)=\frac{1}{2}(\bar{A}-\underline{\mathrm{A}}), \\
A_{d 0} & =\frac{1}{2}\left(\underline{\mathrm{A}}_{d}+\bar{A}_{d}\right), \quad \tilde{A}_{d}=\left(\tilde{a}_{d i j}\right)=\frac{1}{2}\left(\bar{A}_{d}-\underline{\mathrm{A}}_{d}\right), \\
B_{0} & =\frac{1}{2}(\underline{\mathrm{B}}+\bar{B}), \quad \tilde{B}=\left(\tilde{b}_{i j}\right)=\frac{1}{2}(\bar{B}-\underline{\mathrm{B}}), \\
E_{0} & =\frac{1}{2}(\underline{\mathrm{E}}+\bar{E}), \quad \tilde{E}=\left(\tilde{e}_{i j}\right)=\frac{1}{2}(\bar{E}-\underline{\mathrm{E}}),
\end{aligned}
$$

we can rewrite $A, A_{d}, B$ and $E$ as follows:

$$
\left\{\begin{array}{l}
A=A_{0}+A^{\delta}=A_{0}+\sum_{i, j=1}^{n} e_{i} a_{i j}^{\delta} e_{j}^{T},\left|a_{i j}^{\delta}\right| \leq \tilde{a}_{i j}, \\
A_{d}=A_{d 0}+A_{d}^{\delta}=A_{d 0}+\sum_{i, j=1}^{n} e_{i} a_{d i j}^{\delta} e_{j}^{T},\left|a_{d i j}^{\delta}\right| \leq \tilde{a}_{d i j}, \\
B=B_{0}+B^{\delta}=B_{0}+\sum_{i=1}^{n} \sum_{j=1}^{p} e_{i} b_{i j}^{\delta} h_{j}^{T},\left|b_{i j}^{\delta}\right| \leq \tilde{b}_{i j}, \\
E=E_{0}+E^{\delta}=E_{0}+\sum_{i, j=1}^{n} e_{i} e_{i j}^{\delta} e_{j}^{T},\left|e_{i j}^{\delta}\right| \leq \tilde{e}_{i j},
\end{array}\right.
$$

where $e_{k} \in \mathbb{R}^{n}$ or $h_{k} \in \mathbb{R}^{p}$ denotes the column vector with the $k$ th element being 1 and others being 0 .

Remark 1: In practice, the interval uncertainties described in (3) are frequently encountered in many engineering systems, which may result from the variation of operating points, aging of the devices, identification errors, etc. For example, when modeling a real world plant, we often use an interval to estimate a certain parameter so as to allow for some margin for error in the parameter identification. In the past few years, the control problems for systems with interval uncertainty have attracted considerable research attention, see $[11,14]$ and references therein.

In this paper, the nonlinear disturbances are assumed to satisfy the following boundedness condition.

Assumption 1: There exist real constant matrices $G_{1} \in \mathbb{R}^{n \times n}$ and $G_{2} \in \mathbb{R}^{n \times n}$ such that the unknown nonlinear vector function $f(\cdot, \cdot)$ satisfies:

$$
|f(x(t), x(t-\tau))| \leq\left|G_{1} x(t)\right|+\left|G_{2} x(t-\tau)\right| .
$$


Remark 2: The exogenous nonlinear time-varying disturbance has been dealt with in many papers such as [20]. In Assumption 1, the nonlinear disturbance $f(x(t), x(t-\tau))$ in the system (1)-(2) involves the delayed term, which is more general than that studied in [20]. To the best of the authors' knowledge, there has been little research effort reported in the literature on using delay-dependent technique to deal with the robust stabilization problem for stochastic time-delay interval systems with such kind of nonlinear exogenous disturbances.

For the sake of simplicity, we denote:

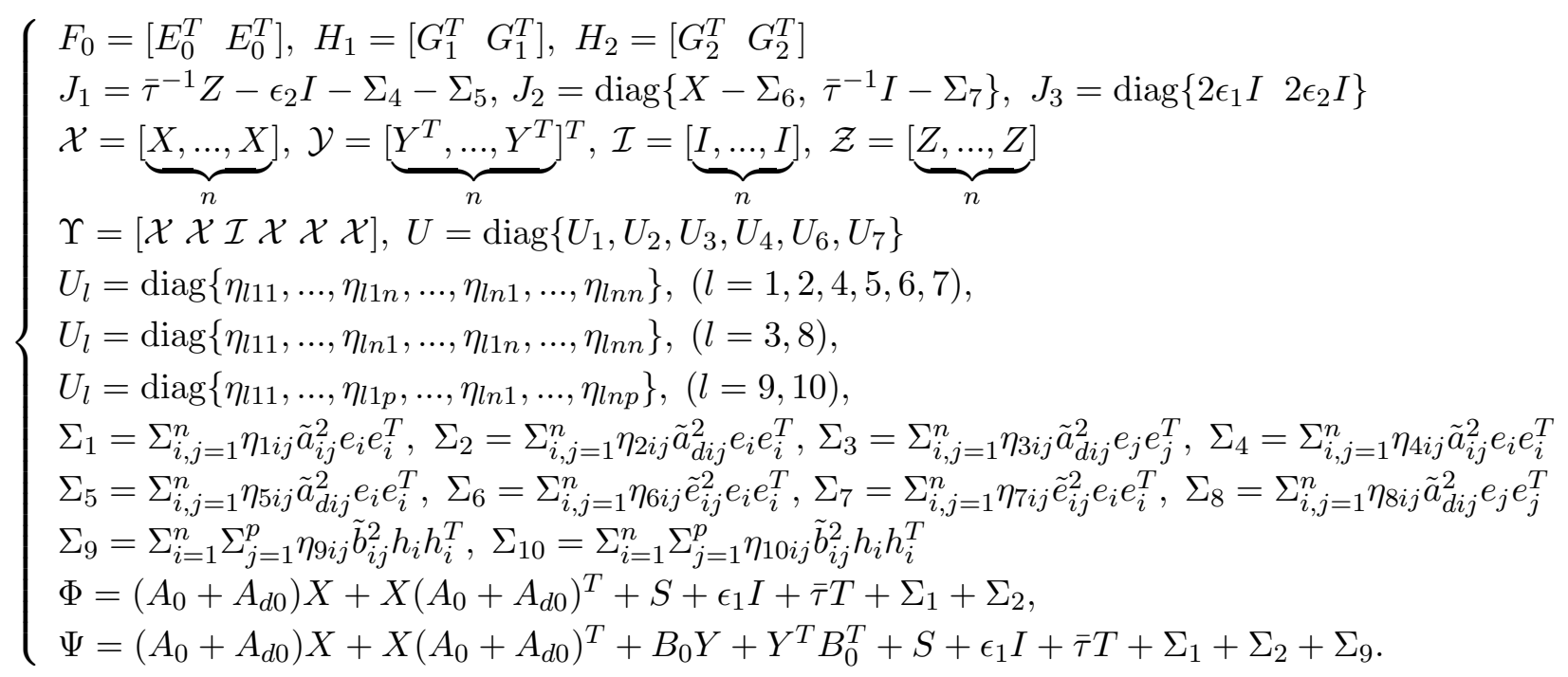

Observe the system (1)-(2) and let $x(t ; \xi)$ denote the state trajectory from the initial data $x(\theta)=\xi(\theta)$ on $-\tau \leq \theta \leq 0$ in $L_{\mathcal{F}_{0}}^{2}\left([-\tau, 0] ; \mathbb{R}^{n}\right)$. Obviously, $x(t, 0) \equiv 0$ is the trivial solution of system (1)-(2) corresponding to the initial data $\xi=0$.

Before formulating the problem to be coped with, we first introduce the following stability concepts for (1)-(2).

Definition 1: For the stochastic time-delay interval system (1)-(2) with $u(t)=0$ and every $\xi \in L_{\mathcal{F}_{0}}^{2}\left([-\tau, 0] ; \mathbb{R}^{n}\right)$, the trivial solution is said to be mean-square asymptotically stable if

$$
\lim _{t \rightarrow \infty} \mathbb{E}|x(t)|^{2}=0
$$

Definition 2: The stochastic time-delay interval system (1)-(2) with the state feedback controller $u(t)=$ $K x(t)$ is said to be robustly stochastically stabilizable if there exists a gain matrix $K \in R^{p \times n}$ such that the closed-loop system is mean-square asymptotically stable.

The purpose of this paper is to design a state feedback controller such that the stochastic time-delay interval system (1)-(2) with nonlinear disturbance is stochastically stabilized by developing delay-dependent techniques.

\section{Robust Stability Analysis}

First, let us give the following lemmas which will be used in the proof of our main results.

Lemma 1: (Schur Complement) Given the constant matrices $\Sigma_{1}, \Sigma_{2}, \Sigma_{3}$ where $\Sigma_{1}=\Sigma_{1}^{T}$ and $0<\Sigma_{2}=\Sigma_{2}^{T}$. Then $\Sigma_{1}+\Sigma_{3}^{T} \Sigma_{2}^{-1} \Sigma_{3}<0$ if and only if

$$
\left[\begin{array}{cc}
\Sigma_{1} & \Sigma_{3}^{T} \\
\Sigma_{3} & -\Sigma_{2}
\end{array}\right]<0
$$


or equivalently,

$$
\left[\begin{array}{cc}
-\Sigma_{2} & \Sigma_{3} \\
\Sigma_{3}^{T} & \Sigma_{1}
\end{array}\right]<0 .
$$

Lemma 2: Let $X, Y, F$ be real matrices of appropriate dimensions with $F^{T} F \leq I$. Then for any scalar $\delta>0$, we have

$$
X F Y+Y^{T} F^{T} X^{T} \leq \delta X X^{T}+\delta^{-1} Y^{T} Y .
$$

Lemma 3: [5] Let $M_{1}, M_{2}, M_{3}$ and $\Xi>0$ be given constant matrices with appropriate dimensions. Then, for any scalar $\varepsilon>0$ satisfying $\varepsilon I-M_{2}^{T} \Xi M_{2}>0$, we have

$$
\left(M_{1}+M_{2} M_{3}\right)^{T} \Xi\left(M_{1}+M_{2} M_{3}\right) \leq M_{1}^{T}\left(\Xi^{-1}-\varepsilon^{-1} M_{2} M_{2}^{T}\right)^{-1} M_{1}+\varepsilon M_{3}^{T} M_{3} .
$$

For presentation convenience, we define the following new state variable

$$
y(t)=A x(t)+A_{d} x(t-\tau)+B u(t)+f(x(t), x(t-\tau)),
$$

and then the systems (1) can be represented as

$$
d x(t)=y(t) d t+E x(t) d \omega(t) .
$$

In the following theorem, a delay-dependent LMI approach is developed to solve the robust stability analysis problem for the stochastic time-delay interval system (1)-(2) with $u(t)=0$, and a sufficient condition is derived ensuring the solvability of the problem.

Theorem 1: Consider the system (1)-(2) with $u(t) \equiv 0$. If there exist positive definite matrices $X>0$, $S>0, Z>0, T>0$ and positive scalars $\epsilon_{1}>0, \epsilon_{2}>0, \eta_{l i j}>0,(i, j=1, \ldots, n, l=1, \ldots, 8)$ such that the following linear matrix inequalities

$$
\left[\begin{array}{ccccccccc}
\Phi & 0 & A_{d 0} & X A_{0}^{T} & X F_{0} & X H_{1} & 0 & \Upsilon & 0 \\
* & -S & 0 & X A_{d 0}^{T} & 0 & 0 & X H_{2} & 0 & \mathcal{X} \\
* & * & -I+\Sigma_{3} & 0 & 0 & 0 & 0 & 0 & 0 \\
* & * & * & -J_{1} & 0 & 0 & 0 & 0 & 0 \\
* & * & * & * & -J_{2} & 0 & 0 & 0 & 0 \\
* & * & * & * & * & -J_{3} & 0 & 0 & 0 \\
* & * & * & * & * & * & -J_{3} & 0 & 0 \\
* & * & * & * & * & * & * & -U & 0 \\
* & * & * & * & * & * & * & * & -U_{5}
\end{array}\right]<0
$$

hold, where $\Phi, F_{0}, H_{1}, H_{2}, J_{1}, J_{2}, J_{3}, \Upsilon, U, U_{5}, U_{8}, \Sigma_{3}, \Sigma_{8}, \mathcal{X}$, and $\mathcal{Z}$ are all defined in (5), then the system (1)-(2) with $u(t) \equiv 0$ is mean-square asymptotically stable.

Proof: Recalling the Newton-Leibniz formula and (7), we can write that, for $t \geq \tau$,

$$
\begin{aligned}
x(t-\tau) & =x(t)-\int_{t-\tau}^{t} d x(s) \\
& =x(t)-\left[\int_{t-\tau}^{t} y(s) d s+\int_{t-\tau}^{t} E x(s) d \omega(s)\right] .
\end{aligned}
$$


Then, it is easy to know from (10) that the following system is equivalent to (1)-(2) with $u(t)=0$ :

$$
\begin{aligned}
d x(t)= & {\left[\left(A+A_{d}\right) x(t)-A_{d} \int_{t-\tau}^{t} y(s) d s-A_{d} \int_{t-\tau}^{t} E x(s) d \omega(s)\right.} \\
& +f(x(t), x(t-\tau))] d t+E x(t) d \omega(t) . \\
x(t)= & \psi(t), \quad t \in[-2 \tau, 0], \quad r(0)=r_{0},
\end{aligned}
$$

where $\psi(t)$ is the initial function. Hence, it suffices to prove the mean-square asymptotic stability of the above system.

Now, let $P=X^{-1}>0, Q=P S P>0, R=Z^{-1}>0$ and define the following Lyapunov-Krasovskii function candidate for the system (11):

$$
\begin{aligned}
V(x(t), t)= & x^{T}(t) P x(t)+\int_{t-\tau}^{t} x^{T}(s) Q x(s) d s+\int_{t-\tau}^{t} \int_{s}^{t} y^{T}(\beta) R y(\beta) d \beta d s \\
& +\int_{t-\tau}^{t} \int_{s}^{t}|E x(\beta)|^{2} d \beta d s .
\end{aligned}
$$

Noticing the fact of

$$
\tau x^{T}(t) W x(t)=\int_{t-\tau}^{t} x^{T}(t) W x(t) d s,
$$

it can be derived by Itô's differential formula [12] that

$$
d V(x(t), t)=\mathcal{L} V(x(t), t) d t+2 x^{T}(t) P E x(t) d \omega(t)
$$

where

$$
\begin{aligned}
\mathcal{L} V(x(t), t)= & x^{T}(t)\left[\left(A+A_{d}\right)^{T} P+P\left(A+A_{d}\right)+Q+E^{T} P E\right] x(t) \\
& -2 x^{T}(t) P A_{d}\left(\int_{t-\tau}^{t} y(s) d s+\int_{t-\tau}^{t} \operatorname{Ex}(s) d \omega(s)\right)+2 x^{T}(t) P f(x(t), x(t-\tau)) \\
& -x^{T}(t-\tau) Q x(t-\tau)+\tau y^{T}(t) R y(t)-\int_{t-\tau}^{t} y^{T}(s) R y(s) d s \\
& +\tau x^{T}(t) E^{T} E x(t)-\int_{t-\tau}^{t}|E x(s)|^{2} d s+\tau x^{T}(t) W x(t)-\int_{t-\tau}^{t} x^{T}(t) W x(t) d s,
\end{aligned}
$$

with $W=P T P>0$.

Noting (4) and Lemma 2, we can calculate that

$$
\begin{aligned}
2 x^{T}(t) P f(x(t), x(t-\tau)) & \leq \epsilon_{1} x^{T}(t) P^{2} x(t)+\epsilon_{1}^{-1} f^{T}(x(t), x(t-\tau)) f(x(t), x(t-\tau)) \\
& \leq \epsilon_{1} x^{T}(t) P^{2} x(t)+\epsilon_{1}^{-1}\left(\left|G_{1 i} x(t)\right|+\left|G_{2 i} x(t-\tau)\right|\right)^{2} \\
& \leq \epsilon_{1} x^{T}(t) P^{2} x(t)+2 \epsilon_{1}^{-1}\left[x^{T}(t) G_{1}^{T} G_{1} x(t)+x^{T}(t-\tau) G_{2}^{T} G_{2} x(t-\tau)\right] .
\end{aligned}
$$

Again, we can obtain from Lemma 2 that

$$
-2 x^{T}(t) P A_{d} \int_{t-\tau}^{t} E x(s) d \omega(s) \leq x^{T}(t) P A_{d} A_{d}^{T} P x(t)+\left|\int_{t-\tau}^{t} E x(s) d \omega(s)\right|^{2} .
$$

Moreover,

$$
\mathbb{E}\left|\int_{t-\tau}^{t} E x(s) d \omega(s)\right|^{2} \leq \int_{t-\tau}^{t} \mathbb{E}|E x(s)|^{2} d s .
$$


Using the Lemma 3 and (6), we have

$$
\begin{aligned}
\tau y^{T}(t) R y(t)= & {\left[A x(t)+A_{d} x(t-\tau)+f(x(t), x(t-\tau))\right]^{T}(\tau R) } \\
& {\left[A x(t)+A_{d} x(t-\tau)+f(x(t), x(t-\tau))\right] } \\
\leq & {\left[A x(t)+A_{d} x(t-\tau)\right]^{T}\left[(\tau R)^{-1}-\epsilon_{2} I\right]^{-1}\left[A x(t)+A_{d} x(t-\tau)\right] } \\
& +\epsilon_{2}^{-1} f^{T}(x(t), x(t-\tau)) f(x(t), x(t-\tau)) \\
\leq & {\left[A x(t)+A_{d} x(t-\tau)\right]^{T}\left[(\tau R)^{-1}-\epsilon_{2} I\right]^{-1}\left[A x(t)+A_{d} x(t-\tau)\right] } \\
& +2 \epsilon_{2}^{-1}\left[x^{T}(t) G_{1}^{T} G_{1} x(t)+x^{T}(t-\tau) G_{2}^{T} G_{2} x(t-\tau)\right] .
\end{aligned}
$$

Substituting (16)-(19) into (15) and taking expectation lead to

$$
\mathbb{E} \mathcal{L} V(x(t), t) \leq \mathbb{E}\left\{\bar{x}^{T}(t) \Omega \bar{x}(t)\right\}+\int_{t-\tau}^{t} \mathbb{E}\left\{\bar{x}^{T}(t, s) \Pi \bar{x}(t, s)\right\},
$$

where

$$
\begin{aligned}
& \left.\Omega:=\left[\begin{array}{cc}
\Omega_{1}+\Delta & 0 \\
0 & \Omega_{2}
\end{array}\right]+\left[\begin{array}{c}
A^{T} \\
A_{d}^{T}
\end{array}\right]\left[(\tau R)^{-1}-\epsilon_{2} I\right)\right]^{-1}\left[\begin{array}{ll}
A & A_{d}
\end{array}\right], \\
& \Pi:=\left[\begin{array}{cc}
-W & -P A_{d} \\
-A_{d}^{T} P & -R
\end{array}\right],
\end{aligned}
$$

with

$$
\begin{aligned}
\bar{x}(t) & =\left[\begin{array}{ll}
x^{T}(t) & x^{T}(t-\tau)
\end{array}\right]^{T}, \bar{x}(t, s)=\left[\begin{array}{ll}
x^{T}(t) & y^{T}(s)
\end{array}\right]^{T}, \\
\Omega_{1} & =\left(A+A_{d}\right)^{T} P+P\left(A+A_{d}\right)+Q+\epsilon_{1} P^{2}+\tau W \\
\Omega_{2} & =2\left(\epsilon_{1}^{-1}+\epsilon_{2}^{-1}\right) G_{2}^{T} G_{2}-Q, \\
\Delta & =P A_{d} A_{d}^{T} P+E^{T} P E+\tau E^{T} E+2\left(\epsilon_{1}^{-1}+\epsilon_{2}^{-1}\right) G_{1}^{T} G_{1} .
\end{aligned}
$$

It remains to show that $\Omega<0$ and $\Pi<0$. By Schur complement lemma, it is easily seen that $\Omega<0$ if and only if

$$
\left[\begin{array}{cccccccc}
\Omega_{1} & 0 & P A_{d} & A^{T} & E^{T} & E^{T} & H_{1} & 0 \\
* & -Q & 0 & A_{d}^{T} & 0 & 0 & 0 & H_{2} \\
* & * & -I & 0 & 0 & 0 & 0 & 0 \\
* & * & * & \epsilon_{2} I-\tau^{-1} Z & 0 & 0 & 0 & 0 \\
* & * & * & * & -P^{-1} & 0 & 0 & 0 \\
* & * & * & * & * & -\tau^{-1} I & 0 & 0 \\
* & * & * & * & * & * & -J_{3} & 0 \\
* & * & * & * & * & * & * & -J_{3}
\end{array}\right]<0,
$$

where $H_{1}, H_{2}$ are defined in (5). 
On the other hand, we note that pre- and post-multiplying (23) by $\operatorname{diag}(X, X, I, I, I, I, I, I)$ yield

$$
\bar{\Omega}=\left[\begin{array}{cccccccc}
\bar{\Omega}_{1} & 0 & A_{d} & X A^{T} & X E^{T} & X E^{T} & X H_{1} & 0 \\
* & -S & 0 & X A_{d}^{T} & 0 & 0 & 0 & X H_{2} \\
* & * & -I & 0 & 0 & 0 & 0 & 0 \\
* & * & * & \epsilon_{2} I-\tau^{-1} Z & 0 & 0 & 0 & 0 \\
* & * & * & * & -X & 0 & 0 & 0 \\
* & * & * & * & * & -\tau^{-1} I & 0 & 0 \\
* & * & * & * & * & * & -J_{3} & 0 \\
* & * & * & * & * & * & * & -J_{3}
\end{array}\right]<0,
$$

with

$$
\bar{\Omega}_{1}=X\left(A+A_{d}\right)^{T}+\left(A+A_{d}\right) X+S+\epsilon_{1} I+\tau T .
$$

Similarly, pre- and post-multiplying $\Pi<0$ by $\operatorname{diag}(X, Z)$ result in

$$
\bar{\Pi}=\left[\begin{array}{cc}
-T & -A_{d} Z \\
-Z A_{d}^{T} & -Z
\end{array}\right]<0 .
$$

Note that we use the shorthand $\mathfrak{M}_{n}\left[\left(M_{1}\right)_{i_{1}, j_{1}},\left(M_{2}\right)_{i_{2}, j_{2}}, \ldots,\left(M_{r}\right)_{i_{r}, j_{r}}\right]$ to represent a $n t h$-order block square matrix whose all nonzero blocks are the $i_{1} j_{1}$ th block $M_{1}$, the $i_{2} j_{2}$ th block $M_{2}, \ldots$, the $i_{r} j_{r}$ th block $M_{r}$, and all other blocks are zero matrices. Then, the matrix $\bar{\Omega}$ can be further rearranged as

$$
\begin{aligned}
& \bar{\Omega}=\left[\begin{array}{cccccccc}
\bar{\Omega}_{10} & 0 & A_{d 0} & X A_{0}^{T} & X E_{0}^{T} & X E_{0}^{T} & X H_{1} & 0 \\
* & -S & 0 & X A_{d 0}^{T} & 0 & 0 & 0 & X H_{2} \\
* & * & -I & 0 & 0 & 0 & 0 & 0 \\
* & * & * & \epsilon_{2} I-\tau^{-1} Z & 0 & 0 & 0 & 0 \\
* & * & * & * & -X & 0 & 0 & 0 \\
* & * & * & * & * & -\tau^{-1} I & 0 & 0 \\
* & * & * & * & * & * & -J_{3} & 0 \\
* & * & * & * & * & * & * & -J_{3}
\end{array}\right] \\
& +\mathfrak{M}_{8}\left[\left(\bar{\Omega}_{1}^{\delta}\right)_{1,1}\right]+\mathfrak{M}_{8}\left[\left(A_{d}^{\delta}\right)_{1,3}, \quad\left(\left(A_{d}^{\delta}\right)^{T}\right)_{3,1}\right] \\
& +\mathfrak{M}_{8}\left[\left(X\left(A^{\delta}\right)^{T}\right)_{1,4}, \quad\left(A^{\delta} X\right)_{4,1}\right]+\mathfrak{M}_{8}\left[\left(X\left(A_{d}^{\delta}\right)^{T}\right)_{2,4}, \quad\left(A_{d}^{\delta} X\right)_{4,2}\right] \\
& +\mathfrak{M}_{8}\left[\left(X\left(E^{\delta}\right)^{T}\right)_{1,5}, \quad\left(E^{\delta} X\right)_{5,1}\right]+\mathfrak{M}_{8}\left[\left(X\left(E^{\delta}\right)^{T}\right)_{1,6}, \quad\left(E^{\delta} X\right)_{6,1}\right] \\
& :=\Phi_{0}+\Phi_{1}+\Phi_{2}+\Phi_{3}+\Phi_{4}+\Phi_{5}+\Phi_{6} \text {, }
\end{aligned}
$$

where

$$
\begin{aligned}
\bar{\Omega}_{10} & =X\left(A_{0}+A_{d 0}\right)^{T}+\left(A_{0}+A_{d 0}\right) X+S+\epsilon_{1} I+\tau T \\
\bar{\Omega}_{1}^{\delta} & =X\left(A^{\delta}+A_{d}^{\delta}\right)^{T}+\left(A^{\delta}+A_{d}^{\delta}\right) X .
\end{aligned}
$$


It follows from Lemma 2 and (3) that, for any real scalars $\eta_{l i j}>0(i, j=1, \ldots, n ; l=1,2)$, the following holds:

$$
\begin{aligned}
\Phi_{1}= & {[X, 0,0,0,0,0,0,0]^{T}\left[\left(A^{\delta}+A_{d}^{\delta}\right)^{T}, 0,0,0,0,0,0,0\right] } \\
& +\left[\left(A^{\delta}+A_{d}^{\delta}\right)^{T}, 0,0,0,0,0,0,0\right]^{T}[X, 0,0,0,0,0,0,0] \\
= & \Sigma_{i, j=1}^{n}\left([X, 0,0,0,0,0,0,0]^{T}\left[\left(e_{i} a_{i j}^{\delta} e_{j}^{T}\right)^{T}, 0,0,0,0,0,0,0\right]\right. \\
& +\left[\left(e_{i} a_{i j}^{\delta} e_{j}^{T}\right)^{T}, 0,0,0,0,0,0,0\right]^{T}[X, 0,0,0,0,0,0,0] \\
& +[X, 0,0,0,0,0,0,0]^{T}\left[\left(e_{i} a_{d i j}^{\delta} e_{j}^{T}\right)^{T}, 0,0,0,0,0,0,0\right] \\
& \left.+\left[\left(e_{i} a_{d i j}^{\delta} e_{j}^{T}\right)^{T}, 0,0,0,0,0,0,0\right]^{T}[X, 0,0,0,0,0,0,0]\right) \\
= & \Sigma_{i, j=1}^{n}\left(\left[e_{j}^{T} X, 0,0,0,0,0,0,0\right]^{T}\left[\left(e_{i} a_{i j}^{\delta}\right)^{T}, 0,0,0,0,0,0,0\right]\right. \\
& +\left[\left(e_{i} a_{i j}^{\delta}\right)^{T}, 0,0,0,0,0,0,0\right]^{T}\left[e_{j}^{T} X, 0,0,0,0,0,0,0\right] \\
& +\left[e_{j}^{T} X, 0,0,0,0,0,0,0\right]^{T}\left[\left(e_{i} a_{d i j}^{\delta}\right)^{T}, 0,0,0,0,0,0,0\right] \\
& \left.+\left[\left(e_{i} a_{d i j}^{\delta}\right)^{T}, 0,0,0,0,0,0,0\right]^{T}\left[e_{j}^{T} X, 0,0,0,0,0,0,0\right]\right) \\
\leq \quad & \Sigma_{i, j=1}^{n}\left(\eta_{1 i j}^{-1}\left[e_{j}^{T} X, 0,0,0,0,0,0,0\right]^{T}\left[e_{j}^{T} X, 0,0,0,0,0,0,0\right]\right. \\
& +\eta_{1 i j} \tilde{a}_{i j}\left[e_{i}^{T}, 0,0,0,0,0,0,0\right]^{T}\left[e_{i}^{T}, 0,0,0,0,0,0,0\right] \\
& +\eta_{2 i j}^{-1}\left[e_{j}^{T} X, 0,0,0,0,0,0,0\right]^{T}\left[e_{j}^{T} X, 0,0,0,0,0,0,0\right] \\
& \left.+\eta_{2 i j} \tilde{a}_{d i j}\left[e_{i}^{T}, 0,0,0,0,0,0,0\right]^{T}\left[e_{i}^{T}, 0,0,0,0,0,0,0\right]\right) \\
= & \mathfrak{M}_{8}\left[\left(\Sigma_{1}+\Sigma_{2}\right)_{1,1}\right]+[\mathcal{X}, 0,0,0,0,0,0,0]^{T} U_{1}^{-1}[\mathcal{X}, 0,0,0,0,0,0,0] \\
& +[\mathcal{X}, 0,0,0,0,0,0,0]^{T} U_{2}^{-1}[\mathcal{X}, 0,0,0,0,0,0,0] \\
= & \mathfrak{M}_{8}\left[\left(\Sigma_{1}+\Sigma_{2}+\mathcal{X} U_{1}^{-1} \mathcal{X}+\mathcal{X} U_{2}^{-1} \mathcal{X}\right)_{1,1}\right] \\
&
\end{aligned}
$$

where $\mathcal{X}, \Sigma_{1}, \Sigma_{2}, U_{1}, U_{2}$ are defined in (5).

Similarly, for any scalars $\eta_{l i j}>0,(i, j=1, \ldots, n, l=3,4,5,6,7,8)$, we have

$$
\begin{aligned}
\Phi_{2} & \leq \mathfrak{M}_{8}\left[\left(\Sigma_{3}\right)_{3,3}\right]+[\mathcal{I}, 0,0,0,0,0,0,0]^{T} U_{3}^{-1}[\mathcal{I}, 0,0,0,0,0,0,0] \\
& =\mathfrak{M}_{8}\left[\left(U_{3}^{-1}\right)_{1,1}, \quad\left(\Sigma_{3}\right)_{3,3}\right] \\
\Phi_{3} & \leq \mathfrak{M}_{8}\left[\left(\Sigma_{4}\right)_{4,4}\right]+[\mathcal{X}, 0,0,0,0,0,0,0]^{T} U_{4}^{-1}[\mathcal{X}, 0,0,0,0,0,0,0] \\
& =\mathfrak{M}_{8}\left[\left(\mathcal{X} U_{4}^{-1} \mathcal{X}\right)_{1,1},\left(\Sigma_{4}\right)_{4,4}\right] \\
\Phi_{4} & \leq \mathfrak{M}_{8}\left[\left(\Sigma_{5}\right)_{4 \times 4}\right]+[0, \mathcal{X}, 0,0,0,0,0,0]^{T} U_{5}^{-1}[0, \mathcal{X}, 0,0,0,0,0,0] \\
& =\mathfrak{M}_{8}\left[\left(\mathcal{X} U_{5}^{-1} \mathcal{X}\right)_{2,2},\left(\Sigma_{5}\right)_{4,4}\right] \\
\Phi_{5} & \leq \mathfrak{M}_{8}\left[\left(\Sigma_{6}\right)_{5 \times 5}\right]+[\mathcal{X}, 0,0,0,0,0,0,0]^{T} U_{6}^{-1}[\mathcal{X}, 0,0,0,0,0,0,0] \\
& =\mathfrak{M}_{8}\left[\left(\mathcal{X} U_{6}^{-1} \mathcal{X}\right)_{1,1},\left(\Sigma_{6}\right)_{5,5}\right] \\
\Phi_{6} & \leq \mathfrak{M}_{8}\left[\left(\Sigma_{7}\right)_{6 \times 6}\right]+[\mathcal{X}, 0,0,0,0,0,0,0]^{T} U_{7}^{-1}[\mathcal{X}, 0,0,0,0,0,0,0] \\
& =\mathfrak{M}_{8}\left[\left(\mathcal{X} U_{7}^{-1} \mathcal{X}\right)_{1,1}, \quad\left(\Sigma_{7}\right)_{6,6}\right] \\
\bar{\Pi} & \leq\left[\begin{array}{cc}
-T & A_{d 0} Z \\
Z A_{d 0}^{T} & -Z+\Sigma_{8}
\end{array}\right]+\left[\begin{array}{c}
0 \\
\mathcal{Z}
\end{array}\right] U_{8}^{-1}\left[\begin{array}{c}
0 \\
\mathcal{Z}
\end{array}\right]^{T},
\end{aligned}
$$

where $\Sigma_{3}, \Sigma_{4}, \Sigma_{5}, \Sigma_{6}, \Sigma_{7}, \Sigma_{8}, U_{3}, U_{4}, U_{5}, U_{6}, U_{7}, U_{8}, \mathcal{I}, \mathcal{Z}$ are defined in (5). 
According to Schur Complement Lemma, after tedious but straightforward calculations, it is followed from the conditions (8), (9) and (24)-(33) that

$$
\bar{\Omega}<0, \bar{\Pi}<0 .
$$

Obviously, from the relationship between $\Omega$ and $\bar{\Omega}$, the relationship between $\Pi$ and $\bar{\Pi}$, and the inequality (20), we can obtain

$$
\Omega<0, \quad \Pi<0
$$

Therefore, we can conclude that

$$
\mathbb{E} \mathcal{L} V(x(t), t)<0,
$$

which indicates that the trivial solution of (11) is asymptotically stability in the mean square. This completes the proof.

Remark 3: In Theorem 1, it is shown that the unforced stochastic time-delay interval system with nonlinear disturbances is mean square asymptotically stable if two LMIs (8) and (9) are feasible, and the stability criteria are dependent on the length of time delay. Note that, by Matlab toolbox, the feasibility of the LMIs (8) and (9) can be checked easily and the maximum allowable bound of the time delay $\tau$ with which the stochastic delayed interval system (1)-(2) is mean square asymptotically stable can be determined.

\section{Delay-Dependent Robust Stabilization}

In this section, we aim to propose a design procedure for the state feedback controller that can robustly stochastically stabilize the addressed stochastic delayed interval systems with nonlinear disturbances. Again, a delay-dependent LMI technique will be developed in order to obtain a less conservative condition. The main result of this paper is given in the following theorem.

Theorem 2: Consider the system (1)-(2). If there exist positive definite matrices $X>0, S>0, Z>0$, $T>0$, a matrix $Y$, and positive scalars $\epsilon_{1}>0, \epsilon_{2}>0, \eta_{l i j}>0,(l=1,2, \ldots, 8), \eta_{9 i m}>0, \eta_{10 i m}>0$ $(i, j=1, \ldots, n ; m=1, \ldots, p)$ such that $(9)$ and the following linear matrix inequality

$$
\left[\begin{array}{ccccccccccc}
\Psi & 0 & A_{d 0} & X A_{0}^{T}+Y^{T} B_{0}^{T} & X F_{0} & X H_{1} & 0 & \Upsilon & 0 & \mathcal{Y}^{T} & \mathcal{Y}^{T} \\
* & -S & 0 & X A_{d 0}^{T} & 0 & 0 & X H_{2} & 0 & \mathcal{X} & 0 & 0 \\
* & * & -I+\Sigma_{3} & 0 & 0 & 0 & 0 & 0 & 0 & 0 & 0 \\
* & * & * & -J_{1}+\Sigma_{10} & 0 & 0 & 0 & 0 & 0 & 0 & 0 \\
* & * & * & * & -J_{2} & 0 & 0 & 0 & 0 & 0 & 0 \\
* & * & * & * & * & -J_{3} & 0 & 0 & 0 & 0 & 0 \\
* & * & * & * & * & * & -J_{3} & 0 & 0 & 0 & 0 \\
* & * & * & * & * & * & * & -U & 0 & 0 & 0 \\
* & * & * & * & * & * & * & * & -U_{5} & 0 & 0 \\
* & * & * & * & * & * & * & * & * & -U_{9} & 0 \\
* & * & * & * & * & * & * & * & * & * & -U_{10}
\end{array}\right]<
$$

hold, where $F_{0}, H_{1}, H_{2}, J_{1}, J_{2}, J_{3}, \Upsilon, U, U_{5}, U_{8}, U_{9}, U_{10}, \Sigma_{3}, \Sigma_{8}, \Sigma_{10}, \mathcal{X}, \mathcal{Y}$ and $\Psi$ are all defined in (5), then with the state feedback controller given by

$$
u(t)=K x(t), \quad K=Y X^{-1},
$$

the closed-loop system is robustly stochastically stable. 
Proof: Applying the controller (35) and Newton-Leibniz formula (10) to the stochastic interval system (1), which is equivalent to replacing $A$ with $A_{c}=A+B K$ in (11), we have

$$
\begin{aligned}
d x(t)= & {\left[\left(A_{c}+A_{d}\right) x(t)-A_{d} \int_{t-\tau}^{t} y(s) d s-A_{d} \int_{t-\tau}^{t} E x(s) d \omega(s)\right.} \\
& +f(x(t), x(t-\tau))] d t+E x(t) d \omega(t), \\
x(t)= & \psi(t), \quad t \in[-2 \tau, 0], \quad r(0)=r_{0} .
\end{aligned}
$$

The Lyapunov-Krasovskii function is chosen as:

$$
\begin{aligned}
V(x(t), t)= & x^{T}(t) P x(t)+\int_{t-\tau}^{t} x^{T}(s) Q x(s) d s+\int_{t-\tau}^{t} \int_{s}^{t} y^{T}(\beta) R y(\beta) d \beta d s \\
& +\int_{t-\tau}^{t} \int_{s}^{t}|E x(\beta)|^{2} d \beta d s .
\end{aligned}
$$

Similar to the proof of Theorem 1, we obtain

$$
\mathbb{E} \mathcal{L} V(x(t), t) \leq \mathbb{E}\left\{\bar{x}(t)^{T} \Gamma \bar{x}(t)\right\}+\int_{t-\tau}^{t} \mathbb{E}\left\{\bar{x}(t, s)^{T} \Pi \bar{x}(t, s)\right\},
$$

where $\Pi$ are defined in $(22)$ and

$$
\Gamma:=\left[\begin{array}{cc}
\Gamma_{1}+\Delta & 0 \\
0 & \Omega_{2}
\end{array}\right]+\left[\begin{array}{c}
A_{c}^{T} \\
A_{d}^{T}
\end{array}\right]\left[(\tau R)^{-1}-\epsilon_{2} I\right]^{-1}\left[A_{c} A_{d}\right],
$$

with

$$
\Gamma_{1}=P\left(A_{c}+A_{d}\right)+\left(A_{c}+A_{d}\right)^{T} P+Q+\epsilon_{1} P^{2}+\tau W
$$

Along the similar line as that in the proof of Theorem 1, we can know from (5), (34) and the expression of $K$ in (35) that

$$
\Gamma<0, \quad \Pi<0
$$

and therefore

$$
\mathbb{E} \mathcal{L} V(x(t), t)<0
$$

which implies that the trivial solution of the closed-loop system (1)-(2) is robustly stochastically stable. The proof is complete.

\section{An Illustrative Example}

In this section, to illustrate the usefulness and flexibility of the theory developed in previous section, we present a simple numerical example. Attention is focused on the design of a stabilizing controller for a class of stochastic time-delay interval system with nonlinear disturbance. 
The system data of (1)-(2) are as follows:

$$
\begin{gathered}
\underline{\mathrm{A}}=\left[\begin{array}{cc}
-3.5 & 0.9 \\
-0.1 & -4.3
\end{array}\right], \quad \bar{A}=\left[\begin{array}{cc}
-2.5 & 1.1 \\
0.1 & -3.7
\end{array}\right], \\
\underline{\mathrm{A}}_{d}=\left[\begin{array}{ll}
1 & 0 \\
0 & 1
\end{array}\right], \quad \bar{A}_{d}=\left[\begin{array}{cc}
1.4 & 0 \\
0 & 1.6
\end{array}\right], \\
\underline{\mathrm{B}}=\left[\begin{array}{cc}
-1.4 & 0 \\
0 & -1.3
\end{array}\right], \quad \bar{B}=\left[\begin{array}{cc}
1.6 & 0 \\
0 & 1.7
\end{array}\right], \\
\underline{\mathrm{E}}=\left[\begin{array}{cc}
0 & -0.1 \\
-0.1 & 0.8
\end{array}\right], \quad \bar{E}=\left[\begin{array}{cc}
2 & 0.1 \\
0.1 & 2.2
\end{array}\right], \\
G_{1}=\left[\begin{array}{cc}
0.5 & 0 \\
0 & 0.1
\end{array}\right], \quad G_{2}=\left[\begin{array}{cc}
0.2 & 0 \\
0 & 0.5
\end{array}\right] .
\end{gathered}
$$

Using Matlab LMI control Toolbox to solve the LMIs (5) and (34), we obtain the maximum allowable bound of the time delay as $\bar{\tau}=2.2793$. Hence, we have the conclusion that the stochastic interval delay system is robustly stabilizable when $\tau \leq 2.2793$.

The solutions of the LMIs (5) and (34) in the case of $\tau=1.0$ are given as follows

$$
\begin{gathered}
X=\left[\begin{array}{ll}
0.8702 & 0.0716 \\
0.0716 & 0.4755
\end{array}\right], \quad S=\left[\begin{array}{cc}
5.4828 & -1.4175 \\
-1.4175 & 2.5971
\end{array}\right], \\
T=\left[\begin{array}{cc}
13.5320 & -0.9107 \\
-0.9107 & 23.2177
\end{array}\right], \quad Z=\left[\begin{array}{cc}
63.9368 & 1.3035 \\
1.3035 & 61.9510
\end{array}\right], \\
Y=\left[\begin{array}{cc}
-27.3075 & -0.9148 \\
-0.8506 & -30.7860
\end{array}\right], \quad K=\left[\begin{array}{cc}
-31.6149 & 2.8373 \\
4.4052 & -65.4085
\end{array}\right], \\
\epsilon_{1}=5.1812, \quad \epsilon_{2}=8.8005, \\
U_{1}=\operatorname{diag}(15.5313,69.7055,69.7725,18.3928), \\
U_{2}=\operatorname{diag}(54.8768,76.5923,76.6807,18.3928), \\
U_{3}=\operatorname{diag}(9.3559,76.6807,76.5923,3.5225), \\
U_{4}=\operatorname{diag}(41.7751,74.7339,74.8157,40.2269), \\
U_{5}=\operatorname{diag}(67.6583,76.6452,76.7313,40.2405), \\
U_{6}=\operatorname{diag}(0.3710,20.6139,20.6253,0.3305), \\
U_{7}=\operatorname{diag}(0.4147,29.0095,29.0219,0.7147), \\
U_{8}=\operatorname{diag}(211.1844,256.0696,481.6419,195.6471), \\
U_{9}=\operatorname{diag}(144.1794,229.0462,158.2998,133.6967), \\
U_{10}=\operatorname{diag}(155.0798,229.0462,158.2998,187.4469) .
\end{gathered}
$$

According to Theorem 2, with the designed controller gain $K$, the closed-loop system is asymptotically stable in the mean square for all admissible interval uncertainties and nonlinear disturbances.

\section{Conclusions}

In this paper, we have investigated the robust stability analysis problem as well as the robust stabilization problem for a class of stochastic time-delay interval systems with nonlinear disturbances. A delay-dependent 
LMI approach has been developed to derive sufficient conditions under which the controlled system is meansquare asymptotically stable, where the conditions are dependent on the length of the time delays. A numerical example has been employed to illustrate the effectiveness of the results obtained.

\section{REFERENCES}

[1] E. K. Boukas and Z. -K. Liu, Deterministic and stochastic time-delay systems, Birkhauser, Boston, 2002.

[2] Y. Cao, J. Lam, L. Hu, Dleay-dependent stochastic stability and $H_{\infty}$ analysis for time-delay systems with Markovian jumping parameters, J. Franklin Institute, Vol. 340, pp. 423-434, 2003.

[3] W. Chen, Z. Guan, X. Liu, Delay-dependent exponential stability of uncertain stochastic systems with mutiple delays:an LMI approach, Systems \& Control Letters, Vol. 54, pp. 547-555, 2005.

[4] Q. Han, Absolute stability of time delay systems with sector-bounded nonlinearity, Automatica, Vol. 41, pp. 2171-2176, 2005.

[5] H. Gao, C. Wang, Delay-Dependent Robust $H_{\infty}$ and $L_{2}-L_{\infty}$ filtering for a class of uncertain nonlineaar time-delay systems, IEEE Trans. Automatic Control, Vol. 48, No. 9, pp. 1661-1666, 2003.

[6] H. Gao, C. Wang, Robust $L_{2}-L_{\infty}$ filtering for uncertain systems with mutiple time-varing state delays, IEEE Trans. Circuits Syst. I, Vol. 50, pp. 594-599, 2003.

[7] H. Gao and T. Chen, New results on stability of discrete-time systems with time-varying state delay, IEEE Trans. Automat. Control, vol. 52, no. 2, pp. 328-334, 2007.

[8] Y. He, M. Wu, J. She, G. Liu, Delay-dependent robust stability criteria for uncertain neutral systems with mixed delays, Systems \& Control Letters, Vol. 51, No.1, pp. 57-65, 2004.

[9] Y. He, M. Wu, J. She and G. Liu, Parameter-dependent Lyapunov functional for stability of time-delay systems with polytopic-type uncertainties, IEEE Trans. Automat. Control, vol. 49, no. 5, pp. 828-832, 2004.

[10] Y. He, Q.-G. Wang, L. Xie and C. Lin, Further improvement of free-weighting matrices technique for systems with timevarying delay, IEEE Trans. Automat. Control, vol. 52, no. 2, pp. 293-299, 2007.

[11] S. Hu and J. Wang, On stabilization of a new class of linear time-invariant interval systems via constant state feedback control, IEEE Trans. Automatic Control, Vol. 45, No. 11, pp. 21.6-2111, 2000.

[12] H. Kushur, Stochastic stability and control, Academic Press, New York, 1976.

[13] X. Mao, J. Lam, S. Xu and H. Gao, Razumikhin method and exponential stability of hybrid stochastic delay interval systems, Journal of Mathematical Analysis and Applications, Vol. 314, No. 1, pp. 45-66, 2006.

[14] X. Mao and J. Chu, Quadratic stability and stabilization of dynamic interval systems, IEEE Trans. Automatic Control, Vol. 48, No. 6, pp. 1007-1012, 2003.

[15] S. Niculescu, E. Verriest, L. Dugard and J. Dion, Stability and robust stability of time-delay systems: a guided tour, In: Stability and control of time-delay systems, L. Duard et al. (ed.) Berlin: Springer, Lect. Notes Control Inf., Sci., 228, pp.1-71., 1998.

[16] P. Shi, M. Mahmoud, S. K. Nguang and A. Ismail, Robust filtering for jumping systems with mode-dependent delays, Signal Processing, Vol. 86, No. 1, pp. 140-152, 2006.

[17] P. Shi, M. Mahmoud, J. Yi and A. Ismail, Worst case control of uncertain jumping systems with multi-state and input delay information, Information Sciences, Vol. 176, No. 2, pp. 186-200, 2005.

[18] H. Shu and G. Wei, $H_{\infty}$ analysis of nonlinear stochastic time-delay systems, Chaos, Solitons E Fractals, Vol. 26, pp. 637-647, 2005.

[19] Z. Wang, F. Yang, D. W. C. Ho and X. Liu, Robust $H_{\infty}$ filtering for stochastic time-delay systems with missing measurements, IEEE Trans. Signal Processing, Vol. 54, No. 7, pp. 2579-2587, 2006.

[20] Z. Wang, J. Lam and X. Liu, Exponential filtering for uncertain Markovian jump time-delay systems with nonlinear disturbances, IEEE Transactions on Circuits and Systems - Part II, Vol. 51, No. 5, pp. 262-268, 2004.

[21] Z. Wang, F. Yang and X. Liu, Robust filtering for systems with stochastic nonlinearities and deterministic uncertainties, Proc. IMechE - Journal of Systems and Control Engineering, Vol. 220, No. 3, pp. 171-182, 2006.

[22] G. Wei, Z. Wang and H. Shu, Nonlinear $H_{\infty}$ control of stochastic delayed systems with Markovian switching, Chaos, Solitons Ef Fractals, in press (doi:10.1016/j.chaos.2006.05.015.)

[23] L. Xie, E. Fridman and U. Shaked, Robust $H_{\infty}$ control of distributed delay systems with application to combustion control, IEEE Trans. Automatic Control, Vol. 46, No. 12, pp. 1930-1935, 2001.

[24] S. Xu, J. Lam, Improved delay-dependent stability results for time-delay systems, IEEE Trans. Automatic Control, Vol. 50, No. 3, pp. 384-387, 2005.

[25] S. Xu, J. Lam, X. Mao, Y. Zou, A new LMI condition for delay-dependent robust stability of stochastic time-delay systems, Asian Journal of Control, Vol. 7, No. 4, pp. 419-423, 2005. 
[26] C. Yuan and X. Mao, Robust stability and controllability of stochastic differential delay equations with Markovian switching, Automatica, Vol. 40, No. 3, pp. 343-354, 2004.

[27] D. Yue and Q.-L. Han, Dleay-dependent exponential stability of stochastic systems with time-varing delay, nonlinearity and Markovian switching, IEEE Trans. Automatic Control, Vol. 50, No.2, pp. 217-222, 2005.

[28] D. Yue and Q.-L. Han, Delayed feedback control of uncertain systems with time-varying input delay, Automatica, Vol. 41, No. 2, pp. 233-240, 2005. 